\title{
Control Mechanism and Security Region for Intentional Islanding Transition
}

Chen, Yu; Xu, Zhao; Østergaard, Jacob

Published in:

PES'09

Link to article, DOI:

10.1109/PES.2009.5276000

Publication date:

2009

Document Version

Publisher's PDF, also known as Version of record

Link back to DTU Orbit

Citation (APA):

Chen, Y., Xu, Z., \& Østergaard, J. (2009). Control Mechanism and Security Region for Intentional Islanding Transition. In PES'09 IEEE. https://doi.org/10.1109/PES.2009.5276000

\section{General rights}

Copyright and moral rights for the publications made accessible in the public portal are retained by the authors and/or other copyright owners and it is a condition of accessing publications that users recognise and abide by the legal requirements associated with these rights.

- Users may download and print one copy of any publication from the public portal for the purpose of private study or research.

- You may not further distribute the material or use it for any profit-making activity or commercial gain

- You may freely distribute the URL identifying the publication in the public portal

If you believe that this document breaches copyright please contact us providing details, and we will remove access to the work immediately and investigate your claim. 


\title{
Control Mechanism and Security Region for Intentional Islanding Transition
}

\author{
Yu Chen, Student Member, IEEE, Zhao Xu, Member, IEEE, and Jacob Østergaard, Member, IEEE
}

\begin{abstract}
This paper investigates the control mechanism for intentional islanding transition, when a Low Voltage (LV) or Medium Voltage (MV) distribution system, which is usually under grid connection mode, is supposed to be separated from the upstream grid, due to either maintenance or a disturbance in the grid. The concept of Islanding Security Region (ISR) has been proposed as an organic composition of the developed control mechanism. The purpose of this control mechanism is to maintain the frequency stability and eventually the security of power supply to the customers, by utilizing resources from generation and demand sides. The control mechanism can be extended to consider the distributed generations like wind power and other innovative technologies such as the Demand as Frequency controlled Reserve (DFR) technique in the future.
\end{abstract}

Index Terms-- control mechanism, distributed generation, frequency control, intentional islanding, security region, stability, wind power

\section{INTRODUCTION}

$\mathrm{T}_{\mathrm{H}}^{\mathrm{H}}$ HE new Danish energy strategy, "A Visionary Danish Energy Policy 2025", published on $19^{\text {th. }}$ January 2007, has an ambitious target to have the share of renewable energy increase to at least $30 \%$ of total national energy consumption by 2025 [1]. This indicates that an essential contribution: approximately $50 \%$ of the electricity demand in Denmark should be supplied by wind energy by 2025 [2], which would encourage more Wind Turbines (WTs) to be installed.

It is well understood that high penetration of wind and other renewable energy will give great challenges to many aspects of operation and control of future power systems. A real life example of this took place at the Danish distribution system on the Bornholm island, which is considered as a

This work was part of project 'NextGen Part 3: Control Architecture for Intentional Islanding Operation in Future Distribution Network with High Penetration of Distributed Generation', and was supported in part by the Danish Transmission System Operator (TSO) Energinet.dk and Technical University of Denmark (DTU).

$\mathrm{Yu}$ Chen is with the Centre for Electric Technology, Department of Electrical Engineering, Technical University of Denmark, Kgs. Lyngby DK2800, Denmark (e-mail: cy@elektro.dtu.dk).

Zhao $\mathrm{Xu}$ is with the Centre for Electric Technology, Department of Electrical Engineering, Technical University of Denmark, Kgs. Lyngby DK2800, Denmark (e-mail: zx@elektro.dtu.dk).

Jacob Østergaard is with the Centre for Electric Technology, Department of Electrical Engineering, Technical University of Denmark, Kgs. Lyngby DK2800, Denmark (e-mail: joe@elektro.dtu.dk). representative of the future power system because of its already very high wind penetration. E.g. in 2007 , the wind energy corresponds to $32.4 \%$ of its electricity demand, compared to $19.7 \%$ in the whole Denmark [3], [4]. On $22^{\text {nd }}$ December 2005, Bornholm was electrically islanded and forced to stay under islanding mode for 51 days, due to the breakdown of its $60 \mathrm{kV}$ interconnection sea cable to the Nordic transmission grid [5]. This accident caused by the ship anchor has endangered the security of power supply on the island, and most WTs had to be shut down during the islanding transition and the following islanded operation period, because the local power stations were unable to adjust production quickly enough to counterbalance the frequency fluctuations caused by WTs [6].

The Bornholm case can be typical for the future and highlight the insufficient frequency regulation capability of today's power system. To enhance the frequency control during islanding operation, both new and old technologies should be utilized. Presently, a promising demand side technology under investigation at the Centre for Electric Technology (CET) at Technical University of Denmark (DTU) is the Demand as Frequency controlled Reserve (DFR) [7], which can assist the frequency regulation, in order to allow for high penetration of wind power under islanding mode. Another relevant technology under development is the WT frequency control [8]. Other useful technologies include but are not limited to load shedding and primary control of synchronous generators. With these technologies, a new control scheme that can enhance frequency control should be developed in order to realize smooth and secure islanding transition, which is the focus of this paper. The new scheme should provide coordinated and coherent control using available resources from both generation and demand sides to ensure secure islanding operation [6].

The paper is organized as follows: Section II analyzes the frequency data acquired by Phasor Measurement Units (PMUs) [9], during a planned islanding period in Bornholm in September 2007, which reveals the difficulty of frequency regulation; Section III proposes a new control mechanism for intentional islanding operation, where the concept of the Islanding Security Region (ISR) is introduced in detail; Section IV presents a study case upon the application of ISR. Finally, Section V concludes the paper and provides the future scope. 


\section{BORNHOLM ISLANDING DATA ANALYSIS}

During the period from $11^{\text {th. }}$ to $14^{\text {th. }}$ September 2007 , the local Distribution System Operator (DSO) Østkraft conducted a planned islanding operation test at Bornholm. The purpose is to collect measurements from different data acquisition systems for the analysis afterwards and to obtain valuable operation experience for future emergency situations where Bornholm has to be islanded. CET has been in charge of data analysis and in our previous work [3], we mainly analyzed the frequency data. We will continue to analyze the voltage data for the islanding period in our future work.

Fig. 1 is the time plot of the PMU measured frequency data during the islanding period where blue and red lines are Bornholm and Nordic frequency respectively during the same period. It is observed in Fig. 1 that during the islanding the Bornholm system frequency fluctuated much more than the Nordic frequency, which has been confirmed by the statistical histograms in Fig. 2, with several high frequency and low frequency spikes.

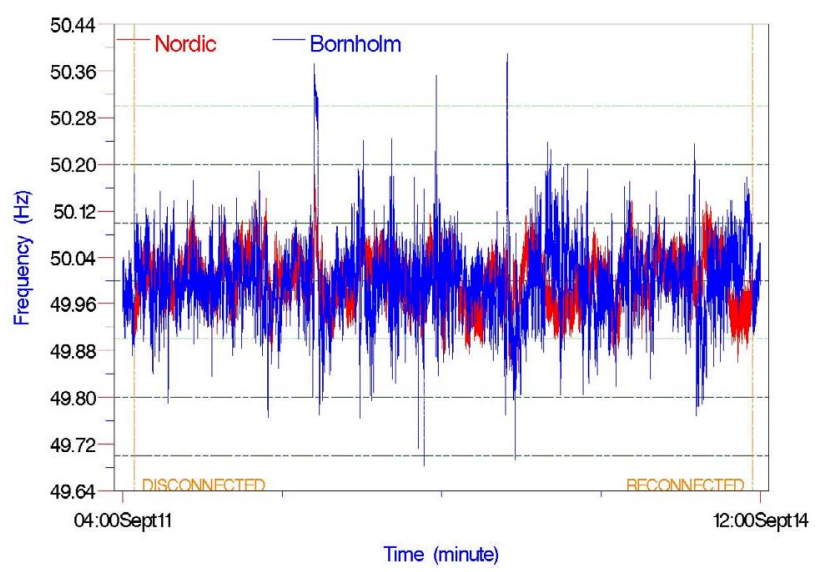

Fig. 1. Time plot of the frequency data during the islanding period

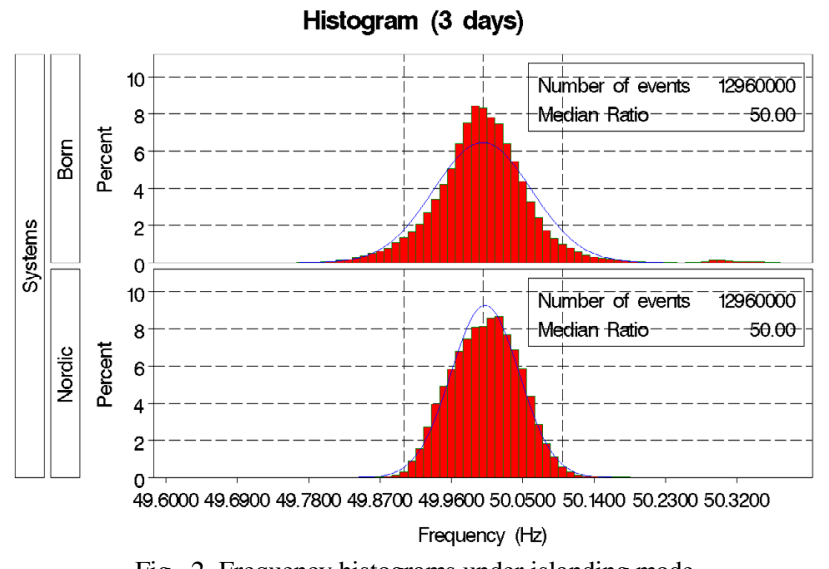

Fig. 2. Frequency histograms under islanding mode

During the transition from grid connection mode to islanding mode, the Bornholm frequency jumped from 49.90 $\mathrm{Hz}$ to around $50.18 \mathrm{~Hz}$ as shown in Fig. 3, even though the islanding operation was well planned beforehand.

During the planned islanding operation, a spare steam plant was initiated before the islanding moment. It gradually increased the power production, in order to decrease the power import through the sea cable. Thus, system can alleviate from the sudden power unbalance pressure at the islanding instant.

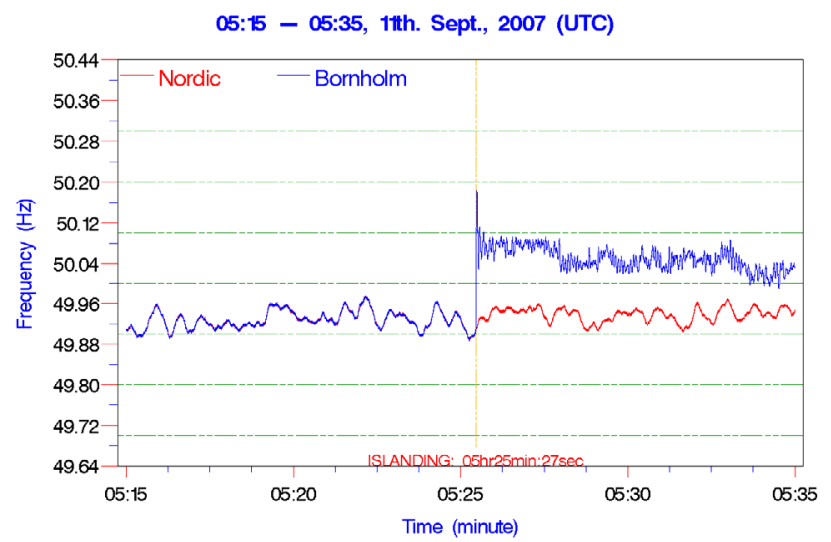

Fig. 3. Time plot of the frequency data during the gird disconnection moment

However, for an accidental islanding event in practice due to e.g. the emergent disturbance from the upstream grid or the breakdown of the interconnection cable, it is hardly possible to have adequate time to adjust the power flow of the cable to the minimum level, as it has been done during this planned islanding operation. Consequently, the chance of islanding induced system blackout can be higher due to e.g. the severe frequency swells or sags at the transition moment. In order to accurately evaluate the security of an islanding operation, particularly in relation to the frequency stability, we propose an ISR concept, based on which a control mechanism for intentional islanding transition is developed. With the ISR concept, the system operator is able to evaluate how far the system is close to the instability in terms of an islanding operation. Correspondingly, proper control and coordination schemes can be taken to best prepare for such operation in advance.

\section{CONTROL MEChANISM}

\section{A. Control mechanism for islanding transition}

As shown in Fig. 4, the proposed control mechanism is applicable for either planned islanding due to maintenance or accidental islanding due to disturbances. Although the control mechanism is developed mainly for islanding operation of a distribution network, it can also be used for system separation of a transmission network. Here we assume that the distribution system, which is going to be islanded, has been identified before islanding operation. It is however, out of the scope of this paper to analyze how to separate a large system into different distribution systems for islanding operation.

There are three stages within the control mechanism: the monitoring, supervision and ISR assessment stage, the control, coordination and ISR re-assessment stage, and the post-islanding transition stage. Under the grid connection mode, the real-time system state, mainly the loads and generations inside the distribution system, should be 
monitored. This system state would then be projected into an already formulated ISR chart for assessment purpose. This ISR is defined as a reference ISR (Ref. ISR) without a control scheme. Meanwhile, the islanding signals or the switching-off signals for the interconnections between the distribution system and upstream grid, sent by the TSO, DSO or relays would be monitored as well. The whole system would either keep its previous state (if the state is within the Ref. ISR) or enter the alarm state (if the state is outside the Ref. ISR), where certain control techniques under grid connection mode can be activated, in order to maintain the system inside the Ref. ISR, if there are no islanding signals. Otherwise, those islanding signals would initiate a corresponding control and coordination scheme, depending on the location of the monitored system state in the Ref. ISR. If the state is inside the Ref. ISR, the distribution system would be stable during the transition and there can be no control or only a moderate control technique involved. If the system state is outside the Ref. ISR, a certain control and coordination scheme should be first searched and established. This is defined as the first stage.

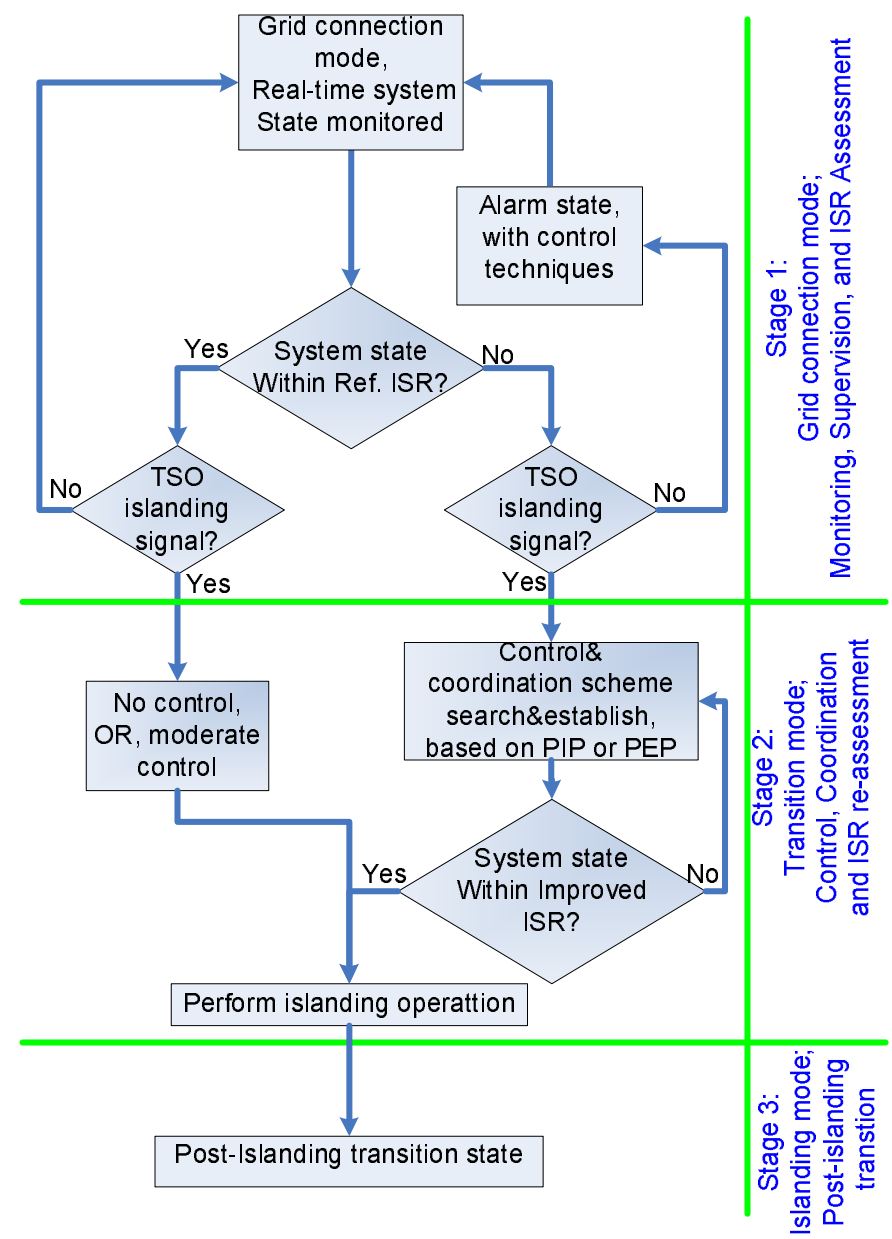

Fig. 4. Control Mechanism for Intentional Islanding Transition

At the second stage, we define two patterns for a control scheme: Power Import Pattern (PIP) and Power Export Pattern (PEP), and under every pattern, the system operator should take different control techniques.

PIP means more loads and less power generation inside the distribution system. To ensure smooth transition, DSO should make coordination to either increase local generations including thermal plants and keep as much wind power as possible, or in the meanwhile, consider demand side techniques, such as load shedding and DFR. On the contrary, PEP means less loads and more generation. Under this situation, the WT frequency control can be implemented, in spite of either decreasing generations or increasing certain loads.

Afterwards, a new improved ISR with this established control scheme should be formulated for re-assessment purpose: is the monitored system state within the new improved ISR? If the system state is within it, islanding operation can be conducted. Otherwise, another control and coordination scheme should be searched and established and the ISR re-assessment repeated.

All in all, the focus of different control techniques is to maintain the power balance of the islanded system within an acceptable range for the transition moment. These control techniques, such as load shedding, droop control for synchronous generations, WT frequency control and DFR should be coordinated in an active and intelligent way. This coordination can be conducted by the application of advanced Information and Communication Technology (ICT), based on IEC 61850 standard communication protocols.

After the control and coordination scheme is implemented and islanding operation is conducted, the system enters the islanding mode, which is the third stage: post-islanding transition state. At this stage, the aim of any control scheme is to maintain and improve the isolated system stability under the islanding mode.

In order to realize the control mechanism, the ideal solution is that there is a central islanding controller inside a distribution system and some other individual controllers of generations and loads involved in the control scheme. The central islanding controller can first locate the system state in the Ref. ISR at the first stage. Besides, it should be able to communicate with the upstream grid. Then the central controller should be able to function as the stage 2 defines. If the system state is within the Ref. ISR and at the same time it receives islanding signals from the upstream grid, the central islanding controller performs the islanding operation by taking no control or a moderate control technique. If the system state is outside the Ref. ISR, the central controller is capable of automatically searching a suitable control scheme and smartly establishing it simultaneously. Then, it formulates a new improved ISR with this specific control scheme, and reassesses whether or not the system state is within the improved ISR. If the state is within the improved ISR this time, the control and coordination scheme signals should be sent to individual controllers of generations and loads by ICT, and then the islanding operation should be conducted. After the implementation of the control scheme, the system enters the third stage, where the concern is about the control under 
islanding mode. Otherwise, another control scheme should be searched and established, and the ISR re-assessment repeated. In a word, the proposed control mechanism includes two threads: the assessment of system state on the one hand (stage 1 and 2), and the coordination and control on the other hand (stage 2 and 3 ).

\section{B. Islanding Security Region}

As we have already mentioned in part A, ISR is the key to the suggested control mechanism, all the following decisions about control schemes should be based on the ISR. This part would explain the ISR mechanism in detail.

Inside a Low Voltage (LV) or a Medium Voltage (MV) distribution system with the possibility to be islanded, many factors can influence the success of the operation. The most important ones are the generations and loads, which are focused herein. There can be different combinations of generations (synchronous generations, WT, etc.) and loads, if both are within their limits. For every combination, islanding operation can be either successful or unsuccessful, depending on the system operation requirements. In this paper, the generations are limited to the traditional generators which can be extended to other distributed ones in the future. The critical combinations of generations and loads are recorded and drawn on the Load-Generation chart. Those recorded combination points can form an ISR. This ISR defines a specific region which can distinguish whether or not the distribution system can meet the operation requirements after being separated from its upstream grid.

If there are no control scheme involved, such as adjustment of generations (decrease/increase of power output of power plants or WTs) and loads (over/under frequency load shedding, DFR), or coordination among generations and loads, the ISR is defined as a reference ISR (Ref. ISR), while it is named an improved ISR if a control scheme is implemented during the transition period.

In practice, DSO or the central islanding controller needs first draw an off-line Ref. ISR for every system/scenario, which has been formed beforehand, and this ISR should cover as many operation states as possible; then, the central islanding controller compares an on-line system state (monitored by PMUs, or other monitoring systems) with this ISR to identify it is inside or outside the ISR. Accordingly, this central controller then makes a decision about how to coordinate and control different elements for the system, if islanding operation is unavoidable at this moment, and formulate an improved ISR for re-assessment as described in part A.

Fig. 5 presents the flow chart for generating the ISR. There are two loops in the flow chart: the first loop or the outer loop for generation change, and the second loop or the inner loop for load change. The main conception is to keep generation fixed first, starting from the system minimum generation level P_gen1, and then increase the loads from the system

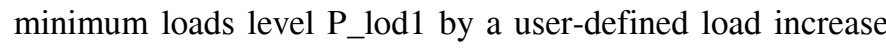
scale y\%, which depends on how much the degree of accuracy is required.

Inside the inner loop, there should be some system operation requirements implemented, which will assess whether the islanding operation is successful or not. Those requirements should depend on the system operator's objectives and different specific systems.

At the very beginning of the study, the authors only consider the frequency requirement, and the islanding operation is considered successful as long as the system frequency can return to the range of $50 \pm 0.2 \mathrm{~Hz}$ within 15 seconds, after the switching-off of the interconnections.

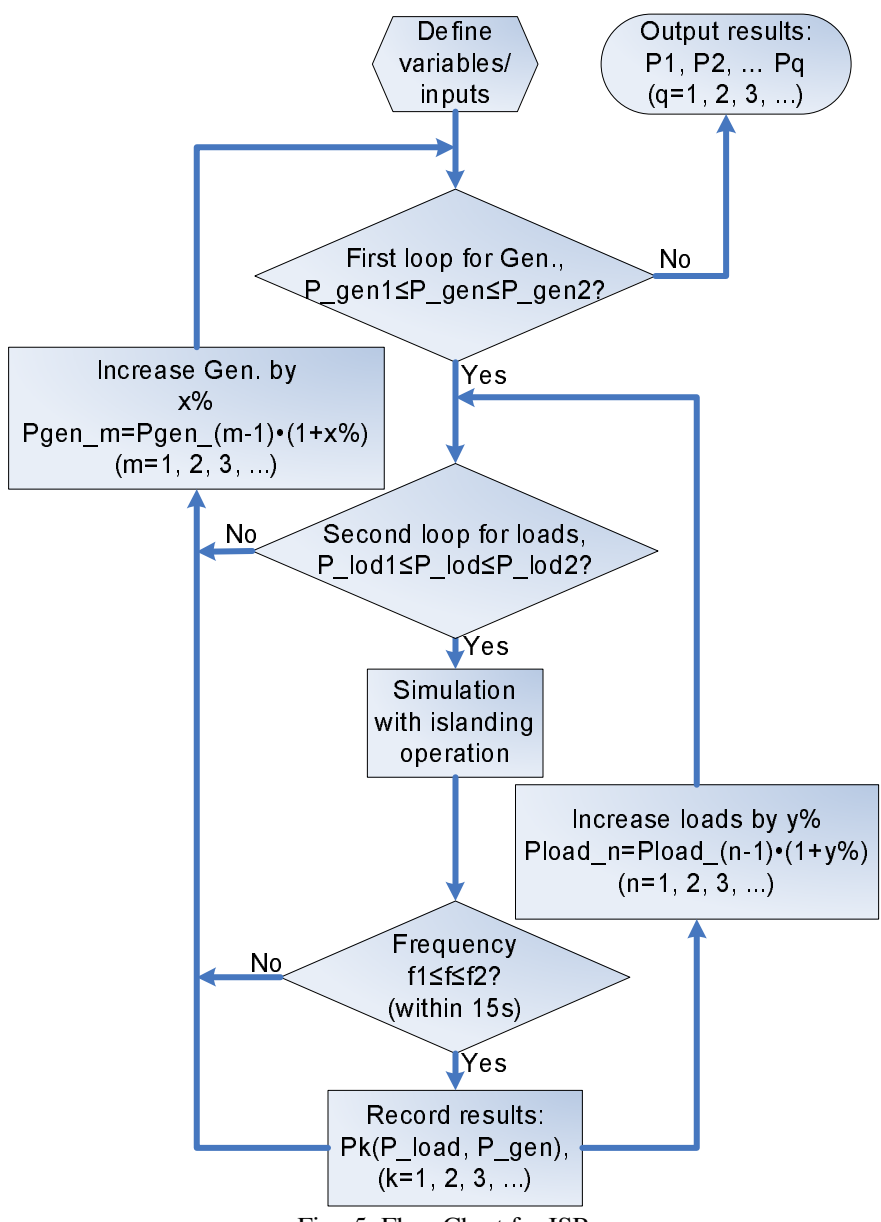

Fig. 5. Flow Chart for ISR

After the whole inner loop, the maximum loads level $\mathrm{P} \_$lod 2 has reached, and the generation should be increased by a defined generation increase scale $\mathrm{x} \%$, similar to the load increase scale $y \%$. And then the second inner loop would repeat.

These two loops should be continuously run until the

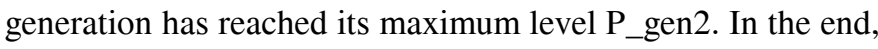
those stable operation points $\mathrm{Pk}$ with corresponding system generations and loads should be recorded, from which the points $\mathrm{Pq}$ with minimum and maximum loads for every generation level would be abstracted. In this way, the ISR can be drawn. 


\section{CASE STUdy}

For simplicity and demonstration purpose, the authors applied the ISR mechanism to a modified 9-bus system in DIgSILENT PowerFactory in the case study. Although the test system is not a distribution network, the application of the ISR islanding mechanism to such a system does not lose its generalities in the context of islanding operation control.

There are some necessary adjustments and assumptions taken in the modified 9-bus system:

1. Another 'Bus 10' was added (rated bus voltage: $230 \mathrm{kV}$ );

2. Another line 'Line 7' between 'Bus 4' and 'Bus 10' was added;

3. 'G1' was substituted by 'External Grid', which was connected to slack bus 'Bus 10'; this 'External Grid' represents the upstream grid;

4. Parameters of generations and loads were changed to their new values, as listed in Table I;

5. 'G2' and 'G3' were equipped with primary controller units and voltage controllers;

6. Loads were independent of frequency and voltage;

7. There were no WTs and DFR;

8. Lumped generations and loads were assumed.

TABLE I

RATED POWER OF GENERATIONS AND LOADS

\begin{tabular}{|c|c|}
\hline Synchronous Generations & Rated Power (MW) \\
\hline G2 & 81.5 \\
\hline G3 & 42.5 \\
\hline & \\
\hline Load & Rated Power (MW) \\
\hline Load A & 50 \\
\hline Load B & 52 \\
\hline Load C & 30 \\
\hline
\end{tabular}

In the modified 9-bus system, shown in Fig. 6, a switching event was defined, which would switch off the added 'Line 7', in order to simulate the islanding action. After the islanding transition, there would be two isolated systems, one is the islanded system, including 8 buses, from 'Bus 2' to 'Bus 9', all generations and loads; the other includes two buses, 'Bus 1 ' and 'Bus 10', and an external grid, which represents the upstream grid.

Based on the explanation and flow chart in part B of section III, the Ref. ISR can be plotted, as shown in Fig. 7. The $\mathrm{X}$-axis $\mathrm{P} \_$lod and $\mathrm{Y}$-axis $\mathrm{P} \_$gen in the chart represent the percentage value of loads to total rated loads and the percentage value of generation to total rated generation in the islanded system, respectively, and three different security regions have been plotted, corresponding to different changeable ranges for both generations and loads, as listed in Table II. Those ranges normally have been already known for a specific distribution system.

As section III explained, the monitored system state would be compared to the Ref. ISR. If the state is inside the region, the islanded system can meet the pre-set requirement after islanding transition, namely, the islanding transition is successful, and the monitored system state is considered stable. If the state is outside the ISR, it indicates that system can not meet the pre-set requirement after the islanding transition, and the state is considered unstable.

In Fig. 7, 'ISR: Curve/3' forms the largest region, which covers all possible stable system states, because the ISR still maintains unchanged, even though the change ranges of both generations and loads are increased. While the smaller region 'ISR: Curve/2' in the middle includes part of those stable states inside 'ISR: Curve/3', because the change ranges of both generations and loads have been shrunk, so does 'ISR: Curve/1', which has the smallest area.



Fig. 6. 10-bus system

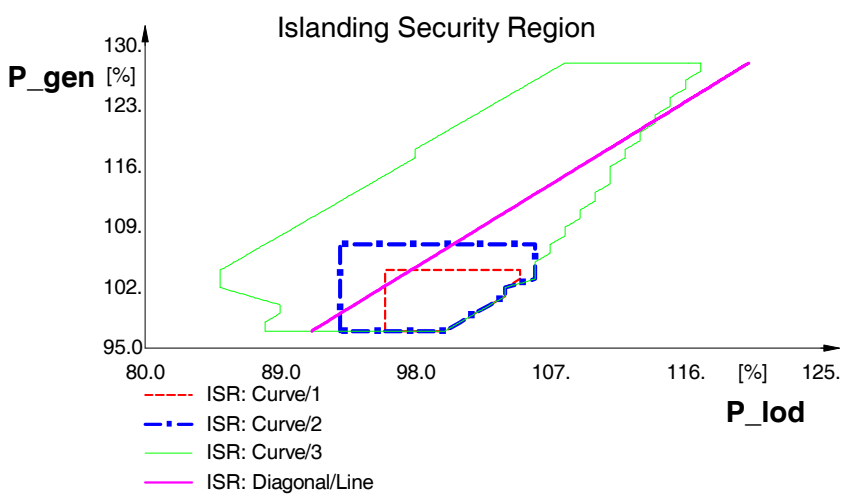

Fig. 7. Security Regions

TABLE II

CHANGEABLE RANGES OF GENERATIONS AND LOADS

\begin{tabular}{|c|c|c|}
\hline Region & P_gen & P_lod \\
\hline ISR: Curve/1 (Red, dashed) & {$[97 \%, 105 \%]$} & {$[97 \%, 105 \%]$} \\
\hline ISR: Curve/2 (Blue, phantom) & {$[97 \%, 108 \%]$} & {$[92 \%, 107 \%]$} \\
\hline ISR: Curve/3 (Green, solid) & {$[97 \%, 129 \%]$} & {$[84 \%, 118 \%]$} \\
\hline
\end{tabular}

Despite those three ISRs, the 'ISR: Diagonal/Line' is another important element in Fig. 7. This line divides the ISR into two regions: the left-top region and the right-down 
region. These two regions correspond to PEP and PIP, respectively, which would lead to different control and coordination scheme, as defined in section III.

\section{CONCLUSIONS}

With the ISR based islanding control mechanism, the system operator can be well informed whether or not a specific distribution system, at any instant, can successfully handle a possible islanding transition, due to either maintenance or a disturbance from the upstream grid. Since wind distributed generations have different characteristics, it is necessary to differentiate them from the traditional generation technology in the islanding control. The ISR mechanism should be extended to include these considerations in the future.

The ISR concept can also provide the operator with a functional tool to effectively assess different control schemes to ensure successful islanding operation. The ultimate goal of the islanding mechanism is to utilize and coordinate those available resources from traditional and innovative techniques, such as load shedding, droop control among power plants, WT frequency control and DFR, in order to maintain real time balance of generation and demand inside the system during the short transition period. Further development of the islanding mechanism is underway to investigate the proper control scheme for coordinated control of different resources to ensure the success of an intentional islanding.

Furthermore, we mainly consider the application of the control mechanism to the frequency stability at present, however, there is a potential to investigate its application to the voltage stability as well, even though the voltage stability is considered fairly localized.

\section{REFERENCES}

[1] The Danish Energy Authority. (2007, Jun.) Energy Policy Statement 2007.[Online].Available:

http://www.ens.dk/graphics/Publikationer/Energipolitik_UK/Energy_polic y_Statement_2007/index.htm

[2] Ea Energy Analyses, "50\% Wind Power in Denmark in 2025 - English Summary," Ea Energy Analyses A/S, July, 2007. [Online]. Available: http://www.ea-

energianalyse.dk/reports/642_50_per_cent_wind_power_in_Danmark_in_ 2025_July_2007.pdf

[3] Y. Chen, Z. Xu, and J. Østergaard, "Frequency Analysis for Planned Islanding Operation in the Danish Distribution System - Bornholm," in Proc. 43rd International Universities Power Engineering Conference, Padova, Italy, 1-4 September 2008.

[4] The Danish Energy Authority. (2008, Apr.) Windturbines - Introduction and Basic Facts. [Online]. Available: http://www.ens.dk/sw14294.asp

[5] ØSTKRAFT Net A/S. (2006.) Årsrapport 2006. [Online]. Available: http://www.oestkraft.dk/download/oestkraftaarsrapport2006.pdf

[6] Edward James-Smith, and Mikael Togeby, "Security of supply for Bornholm-Integration of fluctuating generation using coordinated control of demand and wind turbines-Demand side options for system reserves," September, 2007. [Online]. Available: http://www.eaenergianalyse.dk/reports/640_Security_of_supply_for_Bornholm.pdf

[7] Z. Xu, M. Togeby, and J. Østergaard, "Demand as Frequency Controlled Reserve - Final report of the PSO project," September, 2008. [Online]. Available:http://www.ea- energianalyse.dk/reports/513_demand_as_frequency_controlled_reserve_fi nal_report.pdf

[8] O. Anaya-Lara, F.M. Hughes, N. Jenkins, and G. Strbac, "Contribution of DFIG-based wind farms to power system short-term frequency regulation," in IEE Proc. -Gener. Transm. Distrib., Vol. 153, No. 2, March 2006.

[9] A H. Nielsen, K.O.H. Pedersen, J. Rasmussen, J. Havsager, S. K. Olsen, and P. Jørgensen, "Phasor measurement units in the eastern Danish power system," in CIGRE Conference 2006, Paris, France, pp. 1-5.

\section{BIOGRAPHIES}



Yu Chen (S'08) received the B.Eng. degree in electrical engineering and automation from Nanjing University of Science and Technology (Nanjing, China) in 2004, and the M.Sc. degree in electric power engineering from Chalmers University of Technology (Gothenburg, Sweden) in 2006, respectively. He is currently pursuing his $\mathrm{Ph} . \mathrm{D}$. at the Centre for Electric Technology (CET), Technical University of Denmark (Kgs. Lyngby, Denmark). From September 2005 to May 2006, he conducted his

Master thesis at TST, HVDC Power System department, ABB, Ludvika, Sweden.

His employment experience included the Technology Centre (Beijing, China) in Medium Voltage Products Unit, ABB Asea Brown Boveri Ltd., where he was a $R \& D$ engineer working on electric field simulation.

His current and potential research interests include intentional islanding control and operation and microgrids with high penetration of distributed generations.

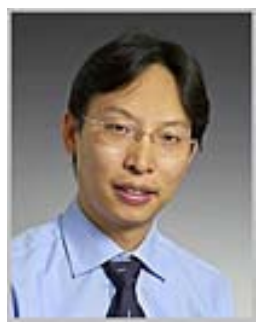

Zhao Xu ((S'00, M'06) received $\mathrm{PhD}$. in electrical engineering from University of Queensland in 2006. $\mathrm{He}$ is now an Assistant Professor at Centre for Electric Technology, Technical University of Denmark. His research interests include demand side, grid integration of wind power, electricity market planning and management, AI applications.

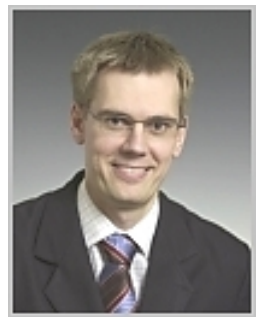

Jacob Østergaard (M'95) is Professor and Head of the Centre for Electric Technology, in the Department of Electrical Engineering, Technical University of Denmark. His research interests include integration of renewable energy, control architecture for future power system, and demand side. Professor Østergaard is serving in several professional organizations including the EU SmartGrids advisory council. 